\title{
Differentially expressed microRNAs in carotid paraganglioma
}

\author{
Anastasiya Snezhkina \\ EIMB RAS, Moscow, Russia \\ leftger@rambler.ru \\ Maria Fedorova \\ EIMB RAS, Moscow, Russia \\ fedorowams@yandex.ru
}

\author{
Elena Pudova \\ EIMB RAS, Moscow, Russia \\ pudova_elena@inbox.ru \\ George Krasnov \\ EIMB RAS, Moscow, Russia \\ gskrasnov@mail.ru
}

\author{
Vladislav Pavlov \\ EIMB RAS, Moscow, Russia \\ vladislav1pavlov@gmail.com \\ Anna Kudryavtseva \\ EIMB RAS, Moscow, Russia \\ rhizamoeba@mail.ru
}

\begin{abstract}
Molecular mechanisms underlying the formation of rare tumors are insufficiently known. It the work, we performed the analysis of microRNA profiles in a rare neuroendocrine tumor of the head and neck, carotid paraganglioma (CPGL). We found four microRNAs (miR-11851/2-3p, miR-431-5p, miR-504-5p, and miR-200c-3p) that are differentially expressed among CPGL samples studied. This is the first study of microRNA expression in CPGL. Obtained results allow a better understanding of molecular changes associated with the development of the tumor.
\end{abstract}

Keywords - carotid paragangliomas, microRNA expression, tumorigenesis

\section{Motivation and aim}

\section{Motivation}

Carotid paraganglioma (CPGL) is a rare neuroendocrine tumor of the head and neck that arises from the microscopic paraganglion at the carotid artery bifurcation. CPGL amounts at more than half of all head and neck paragangliomas (65\%). According to the WHO classification 2017, CPGL is characterized by the variable potential to metastasis; biomarkers of the tumor aggressiveness have not been found. Paragangliomas can occur as both hereditary and sporadic forms. Up to $40 \%$ of paragangliomas are associated with germline mutations in several known genes, predominantly in SDHx [1]. However, due to the rarity of the disease, there are little data on its genetics and molecular mechanisms behind tumor development.

Aim

We perform a complex molecular genetic study of CPGL, including whole exome, transcriptome, microRNA, and methylome analysis [2-4]. The integration of these data allows a better understanding of molecular changes associated with the tumor development and progression, as well as identifying both specific hallmarks of CPGL and common features attributed to all paragangliomas.

\section{Methods}

Total RNA was isolated from 48 tumor samples using the High Pure FFPET RNA Isolation Kit (Roche, Switzerland). MicroRNA libraries were prepared with the NEBNext Multiplex Small RNA Library Prep Kit for Illumina (NEB, USA) according to the manufacturer's protocol. Highthroughput sequencing was performed on the Illumina NextSeq 500 System in the single-end mode with a length of $36 \mathrm{bp}$. Bioinformatics analysis of microRNA data was carried out using the miRge 2.0 pipeline [5].

\section{Results}

We found a list of microRNAs that are differently expressed in CPGL. Four microRNAs (miR-1185-1/2-3p, miR-431-5p, miR-504-5p, and miR-200c-3p) were characterized by the highest variability in the expression among tumor samples studied. All these microRNA were previously shown to be involved in carcinogenesis.

\section{ACKNOWLEDGMENT}

This work was supported by the Russian Science Foundation, grant no. 17-75-20105. The study was performed using the equipment of the EIMB RAS "Genome" center (http://www.eimb.ru/ru1/ckp/ccu_genome_c.php).

\section{REFERENCES}

[1] Zhikrivetskaya S.O. et al. (2017) Molecular markers of paragangliomas/pheochromocytomas. Oncotarget. 8(15): 2575625782.

[2] Snezhkina A.V. et al. (2018) Exome analysis of carotid body tumor. BMC Med Genomics. 11(Suppl 1): 17.

[3] Kudryavtseva A.V. et al. (2019) Mutational load in carotid body tumor. BMC Med Genomics. 12(Suppl 2): 39.

[4] Snezhkina A.V. et al. (2019) Novel potential causative genes in carotid paragangliomas. BMC Med Genetics. 20(Suppl 1): 48.

[5] Lu Y. et al. (2018) miRge 2.0 for comprehensive analysis of microRNA sequencing data. BMC Bioinformatics. 19(1): 275. 